\title{
Sleep-related and non-sleep-related migraine: interictal sleep quality, arousals and pain thresholds
}

\author{
Morten Engstrøm ${ }^{1,2^{*}}$, Knut Hagen ${ }^{1,3}$, Marte Bjørk ${ }^{4,5}$, Gøril Bruvik Gravdahl² and Trond Sand ${ }^{1,2}$
}

\begin{abstract}
Background: The mechanisms associating sleep and migraine are unknown. No previous polysomnographic (PSG) or pain-threshold (PT) study has compared patients with sleep-related migraine attacks (SM), non-sleep related migraine attacks (NSM) and healthy controls.

Methods: We have performed a blinded, prospective exploratory study with case-control design. Thirty-four healthy controls, 15 patients with SM and 18 patients with NSM had interictal PSG heat-, cold- and pressure PT (HPT, CPT, PPT) recordings and completed diary- and questionnaire on sleep and headache related aspects.

Results: NSM patients had more slow-wave sleep (SWS) and more K-bursts than SM patients (K-bursts: $p=0.023$ and SWS: $p=0.030$ ) and controls (K-bursts: $p=0.009$ and SWS: 0.041). NSM patients also had lower HPT and CPT than controls $(p=0.026$ and $p=0.021)$. In addition, SM patients had more awakenings and less $D$-bursts than controls $(p=0.025$ and $p=0.041)$.

Conclusion: SM- and NSM patients differed in objective-, but not subjective sleep quality. NSM patients had PSG findings indicating foregoing sleep deprivation. As foregoing sleep times were normal, a relative sleep deficit might explain reduced PT among NSM patients. The SM patients had signs of slightly disturbed sleep.
\end{abstract}

Keywords: Sleep; Arousal; Migraine; Sleep-related migraine; Non-sleep related migraine; Subjective sleep quality; Polysomnography; Pain thresholds

\section{Background}

Approximately on third (24-42\%) of migraine patients have attacks almost exclusively related to sleep or awakening (sleep migraine = SM) [1,2]. According to ICHD-II SM is not a separate migraine subtype [3], but sleeprelated symptoms are among the most frequently cited trigger factors $[4,5]$. Sleep-related headache is defined by American Academy of Sleep Medicine (AASM) as complaints of headache (either migraine or other types) during sleep or upon awakening [6]. Sleep-related headache may also suggest the presence of an underlying sleep disorder $[7,8]$.

\footnotetext{
* Correspondence: morten.engstrom@ntnu.no

'Department of Clinical Neurosciences, PB 8905, MTFS, Norwegian University of Science and Technology, Trondheim N-7489, Norway

${ }^{2}$ Department of Neurology and Clinical Neurophysiology, St. Olavs Hospital, Trondheim N-7006, Norway

Full list of author information is available at the end of the article
}

Sleep disturbances are commonly reported by migraineurs $[2,9,10]$. However, it is not really known whether migraine attacks are the major cause of the reported sleep disturbances, whether coexistent sleep disturbances trigger migraine attacks during the night or if there are parallel (but non-causal) intrinsic pathophysiological mechanisms linking migraine and sleep problems $[11,12]$. One critical review article suggested that the significance of sleep as a migraine trigger still has not been conclusively determined [13]. Polysomnographic studies have been performed to detect sleep disorders and sleep characteristics in relation to migraine [14-16]; but the results are ambiguous. However, no previous study has compared sleep quality in patients with SM to patients with mainly non-sleep-related migraine attacks (nonsleep-related migraine $=\mathrm{NSM}$ ). This comparison might clarify if disturbed sleep is a factor related specifically to nightly migraine attacks.

\section{实}


Furthermore, the relationship between pain mechanisms and sleep might contribute to explain why some people wake up with migraine. A relationship between sleep and pain has previously been found as sleep loss decrease pain thresholds (PT) in healthy people [17-20]. Disturbed sleep also seems to reduce the descending inhibitory pain control system (DNIC) activity and thereby increasing somatic spontaneous symptoms [21]. Decreased thermal PT (TPT) have also been found 24 hours before migraine attacks [22]. Hence we hypothesized that polysomnographic (PSG)-measures of sleep quality, nightly arousals and PT would be changed among SM patients compared to NSM patients and healthy controls.

Our primary aim of this exploratory, prospective casecontrol study was to compare subjective and objective sleep quality, arousal indices and PT between SM- and NSM patients. The second aim was to compare sleep and pain variables between healthy controls (C) and SMand NSM patients respectively. Thirdly, we wanted to explore the correlation between subjective and objective sleep, PT, and headache severity variables.

\section{Methods}

Participants

Sixty-one migraine patients $(\mathrm{M})$ with and without aura were recruited by advertising in local newspapers. Volunteers called a nurse trained in headache research for a screening interview followed by a consultation with a headache specialist who verified the diagnosis according to ICDH-II [3]. Subjects with two to six migraine attacks per month were selected for the present study. Subjects with coexisting frequent migraine and tension-type headache (TTH), other major health problems (including regular use of neuroleptic-, antiepileptic- or antidepressant drugs, analgesics, or drugs for migraine prophylaxis the last four weeks), or subjects who were pregnant, were not included. Painkillers or triptans for acute migraine were allowed. Based on the headache diary interictal (>two days from attack), patients were selected for the present study and the methodological details have been published previously [23].

The migraineurs were divided in two subgroups according to when the headache usually started. Migraineurs answering either "upon awakening" or "during the night (waking me up)" were defined as having SM, whereas those who answered "during daytime before noon", "during daytime after noon" or "no regular onset time" were defined as having NSM. The pre- and postictal group were too small to qualify for a subgroup analysis. Thirty-three interictal migraineurs SM $(n=15)$ and NSM $(n=18)$ and thirty-four comparable controls according to age and sex were available for the analysis (Table 1). The study was approved by the regional ethics committee and participants signed an informed consent before inclusion.
Table 1 Baseline chracteristics and headache-related data: counts or mean (SD)

\begin{tabular}{|c|c|c|c|}
\hline & $C(n=34)$ & $S M^{1}(n=15)$ & $N S M^{1}(n=18)$ \\
\hline Age (years) & $39.6(13.7)$ & $39.4(14.3)$ & $33.9(11.4)$ \\
\hline Sex: F/M & $20 / 14$ & $10 / 5$ & $15 / 3$ \\
\hline BMI (kg/m2) & $25.3(3.4)$ & $23.6(2.8)$ & $23.4(3.4)^{3}$ \\
\hline $\begin{array}{l}\text { Caffeine beverages } \\
\text { (cups per day) }\end{array}$ & $4.0(3.5)$ & $2.7(2.2)$ & $1.9(2.4)^{4}$ \\
\hline $\begin{array}{l}\text { Alcohol: } 0 \text { (never) to } 5 \\
\text { ( } 4 \text { or more per week) }\end{array}$ & $2.9(1.0)$ & $2.3(1.4)$ & $2.1(1.1)^{4}$ \\
\hline Nicotine: yes/no & $6 / 28$ & $2 / 13$ & $3 / 15$ \\
\hline Physical activity ${ }^{2}$ & $1.8(1.2)$ & $1.6(0.9)$ & $2.1(1.4)$ \\
\hline Days since last menstruation & $18.9(8.6)$ & $16.0(7.4)$ & $13.3(7.9)$ \\
\hline Oral contraceptives: yes/no & $7 / 13$ & $5 / 5$ & $5 / 10$ \\
\hline $\begin{array}{l}\text { Married or common-law } \\
\text { partner/single }\end{array}$ & $25 / 9$ & $7 / 8$ & $12 / 6$ \\
\hline MA/MwoA & & $10 / 5$ & $14 / 4$ \\
\hline Headache time in diary (h/day) & & $1.7(1.5)$ & $2.0(2.3)$ \\
\hline Migraine time in diary (h/day) & & $0.8(1.0)$ & $1.8(2.3)$ \\
\hline Triptan days (0-14) & & $1.9(2.3)$ & $1.5(2.4)$ \\
\hline Analgesic days (0-14) & & $1.1(1.6)$ & $1.9(2.3)$ \\
\hline Headache days last 3 months & & $7.6(7.6)$ & $6.3(3.3)$ \\
\hline Headache intensity (1-4) & & $2.7(0.5)$ & $2.6(0.5)$ \\
\hline Migraine duration (years) & & $24.3(17.7)$ & $16.5(12.0)$ \\
\hline Photophobia (0-2) & & $1.1(0.4)$ & $1.1(0.3)$ \\
\hline Phonophobia (0-2) & & $0.9(0.6)$ & $1.1(0.4)$ \\
\hline
\end{tabular}

Controls (C), SM: Sleep-related migraine attack group, and NSM: none-sleep-related migraine attack group.

'SM and NSM are the corresponding interictal subgroups (background data for interictal PSG and pain threshold variables reported in Table 3).

${ }^{2}(0$ : seldom), 1:1-2x/week, 2:3x/week; sum of exercise and walking to workplace scores.

${ }^{3} \mathrm{NSM}$ or $\mathrm{SM} \neq \mathrm{C}(\mathrm{p}<0.05) .{ }^{4} \mathrm{NSM}$ or $\mathrm{SM} \neq \mathrm{C}(\mathrm{p}<0.01)$.

Pearson chi-square test, Fisher exact test or Mann-Whitney U-test.

Significant differences are emphasized with bold types.

\section{Questionnaires and diaries}

Headache hours per day, average sleep time, sleep latency, long ( $\geq 30 \mathrm{~min})$ and short $(<30 \mathrm{~min})$ awakenings per night were calculated and analyzed from sleep diaries for the 14 days preceding the PSG.

Epworth sleepiness scale (ESS) [24,25], questions adapted from Karolinska sleep questionnaire (KSQ) [26], Pittsburgh sleep quality index (PSQI) [27] and Hospital anxiety and depression subscales (HADS) [28] were administered. The seven PSQI questions (indicating the frequency of common sleep problems; $0-3$ ) were summed into a combined global score variable (PSQIgs, possible range 0-21) [27]. Bothersome tiredness was categorized $(0-4)$ as "none", "less than 7 days per month", "714 days", "> 14 days per month" and "daily". We also had four questions concerning restless legs ("Urge to move the legs", "Rest worsens the urge", "Symptoms improve 
with movement", "Symptoms worsen in the evening or night" [29]). Usual headache intensity, attack length, photophobia, phonophobia and migraine history duration were recorded from semi-structured nurse interviews (Table 1).

\section{PSG}

Patients and controls underwent a full night sleep registration with ambulatory equipment. They slept unattended in our patient-hotel in the neighbor building. PSG was recorded by a Notta recorder (EEG Technology Int.bv, 6092 NM Leveroy, The Netherlands) and analyzed with Stellate Harmonie software (Stellate, Montreal, Quebec, Canada). Eight EEG electrodes were placed according to the International (10-20) system [30] (F3, F4, C3, C4, P3, $\mathrm{P} 4, \mathrm{O} 1, \mathrm{O} 2$ plus $\mathrm{Pz}$ reference and $\mathrm{Cz}$ ground); two electrooculographic electrodes (EOG) applied two $\mathrm{cm}$ lateral and respectively two $\mathrm{cm}$ up and two $\mathrm{cm}$ down from the right and left lateral eye cantus. EOG-reference electrodes were applied to the left (A1) and the right (A2) mastoids. Surface electromyography was registered from submentalis muscles, the left anterior tibialis muscle and trapezius muscle bilaterally.

The following sensors from Breabon Medical Corporation, Ontario, Canada were applied for respiration and circulation measurements: a three-point oronasal airflow thermistor (Airflow temperature sensor R-510), bands around thorax and abdomen to measure respiratory movements (Ultima Respiratory Effort Sensor ${ }^{\mathrm{Tm}}$, piezo- $^{-}$ electric crystals) and a body position sensor (Ultima Body Position Sensor $\left.{ }^{\mathrm{Tm}}\right)$. An infrared index finger oxymeter (model 8000 J3, Nonin Medical Inc, Plymouth, USA) and $10 \mathrm{~mm}$ silver chloride cup ECG electrodes (Natus Medical Inc, San Carlos, USA) were also used. The participants were instructed to go to bed as usual, and write down light-off and light-on times using a synchronized wrist watch.

\section{PSG data analysis}

Analyses were done from noted time for "lights off" in the evening to "lights on" in the morning. Respiratory events were scored automatically and edited visually later. The AASM manual suggest 2 hypopnea definitions based on nasal pressure signals but we had a thermistor. Therefore we chose to analyze hypopneas according to a modified "Chicago criteria" [31] ( $\geq 50 \%$ amplitude reduction or lower amplitude reduction in thermistor signals associated with $4 \%$ desaturation). Automatic periodic leg movement (PLM) analysis was implemented according to the AASM criteria [32]. Manual sleep scoring, arousal scoring and event editing were performed by the first author (specialist in clinical neurophysiology assisted by a sleep expert (last author)). Sleep staging was performed according to "The AASM Manual for the scoring of sleep and associated events" from 2007 [32] with a few exceptions, as described below.

In the present study we wanted to explore fast arousals besides slow arousals as separate events without considering the time interval between the different episodes. Since low frequency episodes have been defined and scored separately as arousals before [33], these D- and K-burst events were used as the "slow counterpart" to the fast AASM-arousals.

First, fast arousals were defined according to the AASM-manual [32] as an abrupt shift of EEG frequency (alpha, theta and/or faster than $16 \mathrm{~Hz}$ activity) lasting 330 seconds, separated with at least 10 seconds of sleep. Arousals were scored in NREM and in REM sleep if associated with increased EMG for more than one second. Although the upper limit for arousal definition is not defined by AASM, we chose to use $30 \mathrm{~s}$ in the present study to avoid ambiguous counts induced by random timing of sleep staging epochs. In this way, an e.g. 25-second EEGfrequency increase will always be counted as one arousal event regardless of its relationship to the epoch boundaries. Therefore, only changes in EEG-activity containing dominating frequencies of $8 \mathrm{~Hz}$ or more lasting more than 30 seconds were classified as an awakening. If a sleep stage N2 K-complex was followed by a high frequency arousal, we scored the arousal without changing the sleep stage to N1.

Secondly, we also scored two additional PSG measures of slow-wave arousal: Delta-bursts (D-bursts), defined as a sequence of delta waves lasting $2 \mathrm{~s}$ or more and exceeding the background amplitude with at least one third [33], and K-bursts, defined as at least two consecutive K-complexes [33]. A K-complex is a negative deflection followed by a positive component with a minimum duration of 0.5 seconds and minimum peak to peak amplitude of $75 \mu \mathrm{V}$. Both fast and slow arousals were scored when observable in at least three out of eight EEG channels. Awakening-, arousal- K- and D-burst- indexes were calculated as event number per sleep hour. Since K- and D-bursts probably reflect similar physiological processes $[34,35]$ they were combined into a KDindex (resembling Cyclic Alternating Pattern (CAP) A1 phase) for correlation analyses in the present paper. The combined KD-index was also chosen rather than the individual $\mathrm{K}$ - and D-burst indexes to reduce the amount of analyses.

\section{Pain thresholds (PT)}

Thermal PT (TPT) and pressure PT (PPT) (algometry) were recorded one hour before the participants had their PSG equipment mounted. Heat and cold PT (HPT and CPT) were measured separately in a fixed order on thenar and the medial forehead on both sides with methods of limits (Senselab - thermotest, thermode area $25 \times 50 \mathrm{~mm}^{2}$, 
Somedic Sales AB, Sweden). Temperature was increased by $1^{\circ} \mathrm{C} / \mathrm{s}$ from a $32^{\circ} \mathrm{C}$ baseline to a $50^{\circ} \mathrm{C}$ maximum for three HPTs followed by three decreasing temperature stimuli to $5^{\circ} \mathrm{C}$ minimum for CPT. PPT were measured at four sites on both sides in a fixed order: $\mathrm{m}$. temporalis $(10 \mathrm{~mm}$ lateral to the external angle of the orbit), m. splenius (C2 level just at the edge of the trapezius muscle about 35-40 $\mathrm{mm}$ lateral to the midline), $\mathrm{m}$. trapezius (10 $\mathrm{mm}$ lateral to the midpoint of a line connecting the acromion and the spinous process of C7) and over distal phalanx middle finger (Algometer type II, probe area $1 \mathrm{~cm}^{2}$, Somedic Sales AB, Sweden). Pressure was increased with $30 \mathrm{kPa} / \mathrm{s}$. Thresholds were repeated three times, left before right, and the average was calculated. All thresholds were measured by one out of two technicians. In subjects who did not feel cold pain at $5^{\circ} \mathrm{C}$, we used the substitution value $4^{\circ} \mathrm{C}$. TPT were expressed as differences from baseline: HPTd (HPT-32) and CPTd (32-CPT) and averaged (right and left sides from all recorded sites) for the present analysis.

\section{Blinding}

For the PSG and PT measurements the technicians were blinded for diagnoses. Scoring of the PSG data was also performed blinded for diagnoses. Two nurses administered the participant appointments and questionnaires. They also accompanied the participants to the technicians after having instructed the participant not to tell anything that could reveal their headache trait or state.

\section{Statistics}

Statistical analyses were performed with PASW statistics v.18 and SYSTAT version 11. Since we found no significant differences in sleep-variables between migraine with and without aura, migraine patients were analyzed as one combined group in the present study.

Two-group comparisons were made by nonparametric Mann-Whitney tests. Categorical data were analyzed with Pearson chi-square test or Fisher's exact test if any cross tabular cells had expected count less than five.

Two-sided p-values less than 0.05 were regarded as significant. No adjustments for multiple comparisons were done because the study was exploratory and we wanted to avoid excessive type II errors and to avoid inappropriately testing of a less relevant universal null-hypothesis [36].

Partial correlation coefficients (adjusted for age) were calculated to explore the association between migraine severity, PT, sleep quality, arousal and sleep symptoms in SM- and NSM patients. Variables were square-root transformed before this calculation.

The power (based on Student's t-tests) to detect large effect sizes equal to 0.8 (and 0.9) SD in two-group comparisons were $78 \%$ (87\%) for C - NSM, 73\% (83\%) for C - SM and $63 \%(73 \%)$ for SM - NSM.

\section{Results}

Baseline characteristics and headache-related data

There were no differences between SM- and NSM patients in baseline characteristics or headache data (Table 1). NSM patients had lower BMI and consumed less caffeine and alcohol than controls, and both NSM- and SM patients had more anxiety symptoms than controls. Triptans were taken within 48 hours before the interictal PSG by two SM- and three NSM patients.

\section{Sleep related symptoms}

Self-reported sleep related symptoms were not significantly different between SM- and NSM patients (Table 2). Both migraine groups reported more subjective sleep problems regarding insomnia, global sleep problems (PSQI) and pain-related sleep difficulties compared to controls. NSM patients were also more often subjectively tired than controls. However, there were no significant differences in hypersomnia as measured by ESS.

\section{Polysomnographic sleep quality, arousals and PT}

NSM patients had more slow wave sleep (SWS, stage N3, $\mathrm{p}=0.023)$, more K-bursts $(\mathrm{p}=0.030)$ and slightly higher nightly mean $\mathrm{SaO} 2$ than SM patients (Table 3). A slightly higher awakening index $(\mathrm{p}=0.025)$, lower D-index $(p=0.04)$ and a tendency to more superficial stage N1 sleep were found among the SM patients compared to controls $(\mathrm{p}=0.05)$. Fast arousal index among the SM patients did not differ from controls either judged by the whole night or separated into specific NREM or REM indexes (not tabulated). There were no significant differences in sleep efficiency, sleep-onset latency, or REM latency.

NSM patients spent more time in bed $(\mathrm{p}=0.041)$, had more stage N3 slow-wave sleep $(p=0.009)$ and a lower index of fast arousals $(\mathrm{p}=0.041)$ than controls. TPT were lower among the NSM patients than controls $(\mathrm{p}=0.026$ and $\mathrm{p}=0.041$ ). PPT also tended to be lower among the NSM patients compared to the SM patients $(\mathrm{p}=0.08)$.

\section{Partial correlations controlled for age in NSM and SM and controls}

Among NSM patients the amount of sleep stage N1 correlated positively with headache frequency (Table 4). The amount of N3 sleep correlated negatively with PPT (Table 4, Figure 1). The amount of KD-bursts correlated negatively with TPT (Figure 2) and headache frequency (Table 4). Among SM patients, the KD-index tended to correlate positively with PPT. The amount of N2 sleep correlated negatively with insomnia and positively with PPT. The amount of sleep stage N3 correlated negatively with headache history duration. Among controls we found no significant correlations in objective versus subjective quality scores or versus PT (not tabulated). 
Table 2 Sleep diary, sleep disorder symptoms, autonomic instability symptoms and emotional state

\begin{tabular}{|c|c|c|c|}
\hline & $C(n=34)$ & $S M(n=15)$ & $\operatorname{NSM}\left(n=18 / 17^{10}\right)$ \\
\hline Average diary sleep time (hour) & $7.3(0.8)$ & $7.2(1.0)$ & $7.3(0.9)$ \\
\hline Long awakenings in diary ${ }^{1}$ (no) & $0.1(0.2)$ & $0.2(0.2)$ & $0.3(0.5)$ \\
\hline Short awakenings in diary ${ }^{2}$ (no) & $0.2(0.2)$ & $0.2(0.5)$ & $0.3(0.5)$ \\
\hline Sleep latency in diary ${ }^{3}$ & $0.4(0.4)$ & $0.7(0.8)$ & $0.5(0.5)$ \\
\hline Epworth sleepiness scale (0-24) & $5.6(3.1)$ & $5.5(3.3)$ & $7.2(4.7)$ \\
\hline Snoring/apnea KSQ score (0-8) & $1.7(1.6)$ & $1.5(1.3)$ & $1.6(1.8)$ \\
\hline Daytime tiredness frequency (0-4) & $0.7(0.8)$ & $1.1(1.0)$ & $1.3(1.0)^{12}$ \\
\hline Insomnia KSQ score $(0-16)^{4}$ & $3.4(2.3)$ & $6.3(3.7)^{12}$ & $5.8(2.8)^{12}$ \\
\hline PSQlgs $(0-21)^{5}$ & $3.8(2.6)$ & $6.5(3.1)^{12}$ & $5.9(3.5)^{11}$ \\
\hline Pain-related sleep trouble $(1-4)^{6}$ & $1.3(0.7)$ & $1.8(1.0)^{11}$ & $2.0(1.1)^{12}$ \\
\hline Restless legs $(0-1)^{7}$ & $0.1(0.4)$ & $0.3(0.5)$ & $0.3(0.5)$ \\
\hline Depression score $(0-21)^{8}$ & $1.6(2.1)$ & $2.1(2.1)$ & $2.9(2.8)$ \\
\hline Anxiety score $(0-21)^{8}$ & $2.9(2.6)$ & $5.3(2.6)^{12}$ & $6.0(3.3)^{12}$ \\
\hline Autonomic index $(0-30)^{9}$ & $1.5(1.4)$ & $5.7(5.0)^{13}$ & $6.3(3.3)^{13}$ \\
\hline
\end{tabular}

Controls (C), SM: Sleep-related migraine attack subgroup. NSM: non-sleep-related migraine.

${ }^{1}$ Awakenings lasting more than 30 minutes.

${ }^{2}$ Awakenings lasting less than 30 minutes.

${ }^{3}$ Categorized as 0: <15 min, 1: 15-30 min, 2: 30-90 min, 3: > $90 \mathrm{~min}$.

${ }^{4}$ Sum of four insomnia-questions in KSQ (Karolinska sleep questionnaire).

${ }^{5}$ Pittsburgh sleep quality index.

${ }^{6}$ frequency of pain-related sleep problems during the last month from the PSQI questionnaire. ${ }^{7}$ Restless legs $(1=$ answered yes to all four obligatory criteria).

${ }^{8}$ Sum of seven questions about depression and anxiety symptoms respectively during the last week from the Hospital Anxiety and Depression Scale

(HADS) questionnaire.

${ }^{9}$ Sum of 10 questions about palpitations, nausea, unsteadiness, muscle weakness, tremulousness, dizziness, blurred vision, shivering, and paleness.

${ }^{10}$ Diary data lacking from one NSM subject.

${ }^{11} \mathrm{NSM}$ or $\mathrm{SM} \neq \mathrm{C}(\mathrm{p}<0.05) .{ }^{12} \mathrm{NSM}$ or $\mathrm{SM} \neq \mathrm{C}(\mathrm{p}<0.01) .{ }^{13} \mathrm{NSM}$ or $\mathrm{SM} \neq \mathrm{C}(\mathrm{p}<0.001)$. (Mann-Whitney U-test). Significant differences are emphasized with bold types.

\section{Discussion}

In this first prospective exploratory case-control study evaluating NSM- and SM patients and controls, we found that NSM had increased objective sleep quality, increased subjective daytime tiredness and reduced PT. The SM patients on the other side had signs of reduced objective sleep quality, but had normal PT and did not report increased subjective daytime tiredness.

\section{NSM}

NSM patients had increased daytime tiredness, more SWS (N3) sleep, less fast arousals and a higher K-burst index than controls. After sleep deprivation in healthy controls increased amount of K-complexes [37] and SWS $[37,38]$ and reduced amount of fast arousals [38] are found. However, based on the sleep diaries the NSM patients had the same sleep times as the SM patients and controls. A higher need of sleep and a relative sleep deprivation among the NSM patients could be one explanation. Another explanation could be hypoarousability, i.e. hypofunction of the CNS arousal system [14].

The lower caffeine intake among NSM patients can possibly be related to the daytime tiredness. However, reduced caffeine intake is unlikely to explain our PSG findings among NSM patients because a low-to-moderate amount of caffeine has been found to have little effect on subjective and objective sleep apart from more stage I sleep (Rechtschaffen and Kales) in insomniacs [39].

We also found reduced TPT among the NSM patients. Reduced PT has previously been found among sleepdeprived healthy persons [17-20]. In addition, EEG has in several old studies been found to resemble drowsiness in several migraineurs [40]. These observations do also fit with our interpretation of the present data, i.e. that the NSM patients may be hypoaroused and suffer from (relative) sleep deprivation.

Moreover, there were negative correlations between N3 sleep and PPT and between KD-bursts and TPT among the NSM patients. Slow bursts are said to reflect the slow wave sleep propensity, occurring with highest frequency before SWS in the first sleep cycles [41]. Hence, increased SWS and slow bursts could indicate a higher need for sleep in NSM patients compared to SM patients. Our findings do also suggest a possible dose-response relationship between the proposed sleep deprivation and the reduced PT.

\section{SM}

The SM patients had no signs consistent with sleep deprivation (e.g. increased daytime tiredness and reduced 
Table 3 PSG sleep quality, and pain threshold mean values (SD) for controls, and interictal migraine subgroups

\begin{tabular}{|c|c|c|c|}
\hline & $C(n=34)$ & $S M(n=15)$ & $\operatorname{NSM}(n=18)$ \\
\hline Time in bed & $453(58)$ & $465(57)$ & $488(46)^{4}$ \\
\hline Total sleep time (min) & $409(68)$ & $417(67)$ & $451(52)$ \\
\hline Sleep efficiency (\%) & $90.0(8.1)$ & $89.4(7.6)$ & $92.4(4.2)$ \\
\hline Latency to sleep onset (min) & $12.8(14.6)$ & $13.8(25.1)$ & $7.4(7.7)$ \\
\hline Awakening index (no/h) & $0.99(0.59)$ & $1.45(0.84)^{4}$ & $1.12(0.63)$ \\
\hline Wake after sleep onset (min) & $30.9(26.7)$ & $34.5(19.0)$ & $29.5(16.4)$ \\
\hline Stage N1 (min) & $27(19)$ & $35(17)^{6}$ & $29(13)$ \\
\hline Stage N2 (min) & $197(47)$ & $194(44)$ & $206(45)$ \\
\hline Stage N3 (min) & $86(31)$ & $88(25)^{3}$ & $104(28)^{3,5}$ \\
\hline REM (min) & $99(26)$ & $99(38)$ & $112(32)$ \\
\hline $\mathrm{SaO} 2$ mean (\%) & $95.2(1.4)$ & $95.2(1.4)^{3}$ & $96.1(1.0)^{3,4}$ \\
\hline Apnea-hypopnea index (per hour) & $2.7(3.3)$ & $2.6(2.6)$ & $2.2(3.3)$ \\
\hline Periodic limb movement index (per hour) & $6.7(10.3)$ & $4.0(6.3)$ & $8.4(11.4)$ \\
\hline Fast arousal index (per sleep hour) & $18.3(5.7)$ & $17.4(8.6)$ & $15.5(9.7)^{4}$ \\
\hline Fast arousal index (per hour REM sleep) & $21.8(8.7)$ & $21.3(11.3)$ & $18.2(14.6)$ \\
\hline KD-burst index (per sleep hour) & $14.8(10.9)$ & $9.6(7.3)$ & $15.4(9.8)$ \\
\hline D-burst index (per sleep hour) & $11.8(8.0)$ & $7.3(5.7)^{4}$ & $11.3(8.3)$ \\
\hline K-burst index (per sleep hour) & $3.0(3.8)$ & $2.4(2.2)^{3}$ & $4.0(2.5)^{3,4}$ \\
\hline PPTavg $^{1}(\mathrm{kPa})$ & $661(249)$ & $586(141)$ & $519(125)^{6}$ \\
\hline $\mathrm{HPTavg}^{2}\left({ }^{\circ} \mathrm{C}\right)$ & $13.6(3.1)$ & $12.6(3.3)$ & $11.2(3.7)^{4}$ \\
\hline $\mathrm{CPTavg}^{2}\left({ }^{\circ} \mathrm{C}\right)$ & $20.7(6.3)$ & $18.3(6.3)$ & $16.1(7.4)^{4}$ \\
\hline
\end{tabular}

C: Controls, SM: Sleep-related migraine attack subgroup and NSM: non-sleep-related migraine recorded in an interictal phase. PPT: pressure pain threshold, HPT: Heat pain threshold, CPT: cold pain threshold. avg: Regional averages from either ${ }^{1}$ splenius, trapezius, temporalis, and index finger or ${ }^{2}$ forehead and palm. HPT and CPT are expressed as differences from the $32^{\circ} \mathrm{C}$ baseline.

Mann-Whitney U-test: ${ }^{3} \mathrm{NSM} \neq \mathrm{SM}(\mathrm{p}<0.05) .{ }^{4} \mathrm{NSM}$ or $\mathrm{SM} \neq \mathrm{C}(\mathrm{p}<0.05) .{ }^{5} \mathrm{NSM} \neq \mathrm{C}(\mathrm{p}<0.01),{ }^{6} \mathrm{NSM}$ or $\mathrm{SM} \neq \mathrm{C}(\mathrm{p}=0.05)$. One control and two NSM subjects were excluded from the PLM analysis and one SM from arousal analysis because of loosened electrodes. Significant differences are emphasized with bold types.

PT) even though we found signs of disturbed sleep compared to controls. Age-adjusted headache duration in years was related to a distinct reduction of SWS among SM patients. Also, increasing insomnia symptoms were mainly related to reduced N2 sleep and there was an association between reduced N2 sleep and reduced PT. Furthermore SM patients also had fewer K-bursts than NSM patients. In coherence with findings of Della Marca [14] SM patients also had fewer D-bursts than controls. Slow bursts are frequent before and during SWS [41]. If slow bursts can be interpreted as a measure of the ability to get enough SWS, it also is consistent with a reduced ability to achieve sufficient SWS among SM patients. N2 sleep then might partially compensate for a possible lack of SWS in SM patients.

Nightly hypoxia is related to headache and tiredness [11] and SM patients had a slightly lower mean $\mathrm{SaO} 2$ during the whole night than NSM patients. However, mean $\mathrm{SaO} 2$ did not differ in SM patients compared to controls, and we could not detect any significant difference in the apnea- hypopnea index between these groups.
What is the importance of headache onset time?

In our first paper from this study [23], interictal migraineurs had increased amount of awakenings during sleep, but paradoxically tended to have less fast arousals and more slow wave sleep. From the present results we can see that the explanation is subgroup differences. The SM patients had increased awakenings and the NSM patients had increased slow wave sleep and less fast arousals.

A migraine attack may be interpreted as an example of genetically determined adaptive behavioral response to internal or external stressors that it is orchestrated by a threatened brain [42]. Also in the present study both SM and NSM had increased "load" with more symptoms of sleep disturbances and anxiety compared to controls $[43,44]$. Increased neural activity can increase sleep need [45], but we could not detect differences in work frequency, physical activity, affective or sleep symptoms between the NSM and SM groups.

Why were the SM patients not equally or more tired than the NSM patients? In line with Cortelli [42] we found signs of slightly disturbed sleep among SM patients 
Table 4 Partial age-adjusted correlations ${ }^{1}$ between sleep, symptoms, pain thresholds, among interictal migraineurs subgroups

\begin{tabular}{|c|c|c|c|c|c|c|c|c|}
\hline \multirow[b]{2}{*}{ NSM $(n=18)$} & \multicolumn{2}{|c|}{ Sleep quality } & \multicolumn{3}{|c|}{ Pain thresholds } & \multicolumn{3}{|c|}{ Headache } \\
\hline & Insomnia $^{2}$ & $\mathrm{PSQI}^{3}$ & PPT & HPT & CPT & Headache days ${ }^{4}$ & Headache intensity (1-4) & Headache duration (years) ${ }^{1}$ \\
\hline Sleep N1 & 0.12 & 0.41 & 0.16 & 0.02 & 0.16 & $0.60^{*}$ & 0.21 & 0.35 \\
\hline Sleep N2 & 0.18 & 0.26 & 0.29 & -0.05 & -0.04 & 0.18 & 0.08 & -0.11 \\
\hline Sleep N3 & -0.40 & $-0.47\left(^{*}\right)$ & $-0.50^{*}$ & -0.18 & -0.21 & -0.24 & 0.03 & -0.23 \\
\hline REM sleep & -0.15 & -0.25 & -0.04 & 0.08 & -0.27 & 0.21 & 0.19 & 0.03 \\
\hline Fast arousal index & $0.46\left(^{*}\right)$ & $0.45\left(^{*}\right)$ & 0.30 & -0.19 & -0.23 & -0.34 & -0.10 & 0.09 \\
\hline $\begin{array}{l}\text { Slow arousal (KD-) } \\
\text { index }\end{array}$ & 0.13 & -0.33 & 0.05 & $-0.57^{*}$ & $-0.57^{*}$ & $-0.64 * *$ & -0.01 & $-0.46\left(^{*}\right)$ \\
\hline $\operatorname{SM}\left(n=15^{5}\right)$ & Insomnia $^{2}$ & $\mathrm{PSQI}^{3}$ & PPT & HPT & CPT & Headache days ${ }^{4}$ & Headache intensity (1-4) & duration (years) \\
\hline Sleep N1 & 0.32 & 0.28 & 0.07 & -0.12 & 0.08 & 0.11 & 0.19 & -0.13 \\
\hline Sleep N2 & $-0.69 * *$ & $-0.46(*)$ & $0.54^{*}$ & -0.20 & 0.08 & $-0.50\left(^{*}\right)$ & 0.04 & 0.28 \\
\hline Sleep N3 & 0.41 & 0.33 & -0.36 & -0.11 & -0.44 & 0.39 & -0.22 & $-0.63^{*}$ \\
\hline REM sleep & -0.13 & -0.11 & 0.15 & 0.10 & 0.34 & -0.40 & -0.01 & -0.06 \\
\hline Fast arousal index & 0.30 & 0.26 & 0.15 & -0.21 & -0.08 & -0.02 & 0.18 & $-0.53\left(^{*}\right)$ \\
\hline $\begin{array}{l}\text { Slow arousal (KD-) } \\
\text { index }\end{array}$ & -0.36 & -0.01 & $0.53(*)$ & -0.19 & -0.31 & 0.05 & 0.40 & -0.12 \\
\hline
\end{tabular}

NSM: Non-sleep related migraine. SM: sleep related migraine.

${ }^{1}$ All variables were square root transformed before correlation was computed. SM: Sleep-related migraine attack subgroup and NSM: non-sleep-related migraine recorded in an interictal phase. PPT: pressure pain threshold, HPT: Heat pain threshold, CPT: cold pain threshold.

${ }^{2}$ Sum of four insomnia-questions in KSQ (Karolinska sleep questionnaire).

${ }^{3}$ Pittsburgh sleep quality index frequency of pain-related sleep problems during the last month from the PSQI questionnaire.

${ }^{4}$ Headache days last three months.

${ }^{5}$ One SM excluded from fast and slow arousal scoring because of loosened electrode.

$\left.\mathrm{p}<0.10{ }^{*}\right), \mathrm{p}<0.05^{*}, \mathrm{p}<0.01^{* *}$ Significant differences are emphasized with bold types.
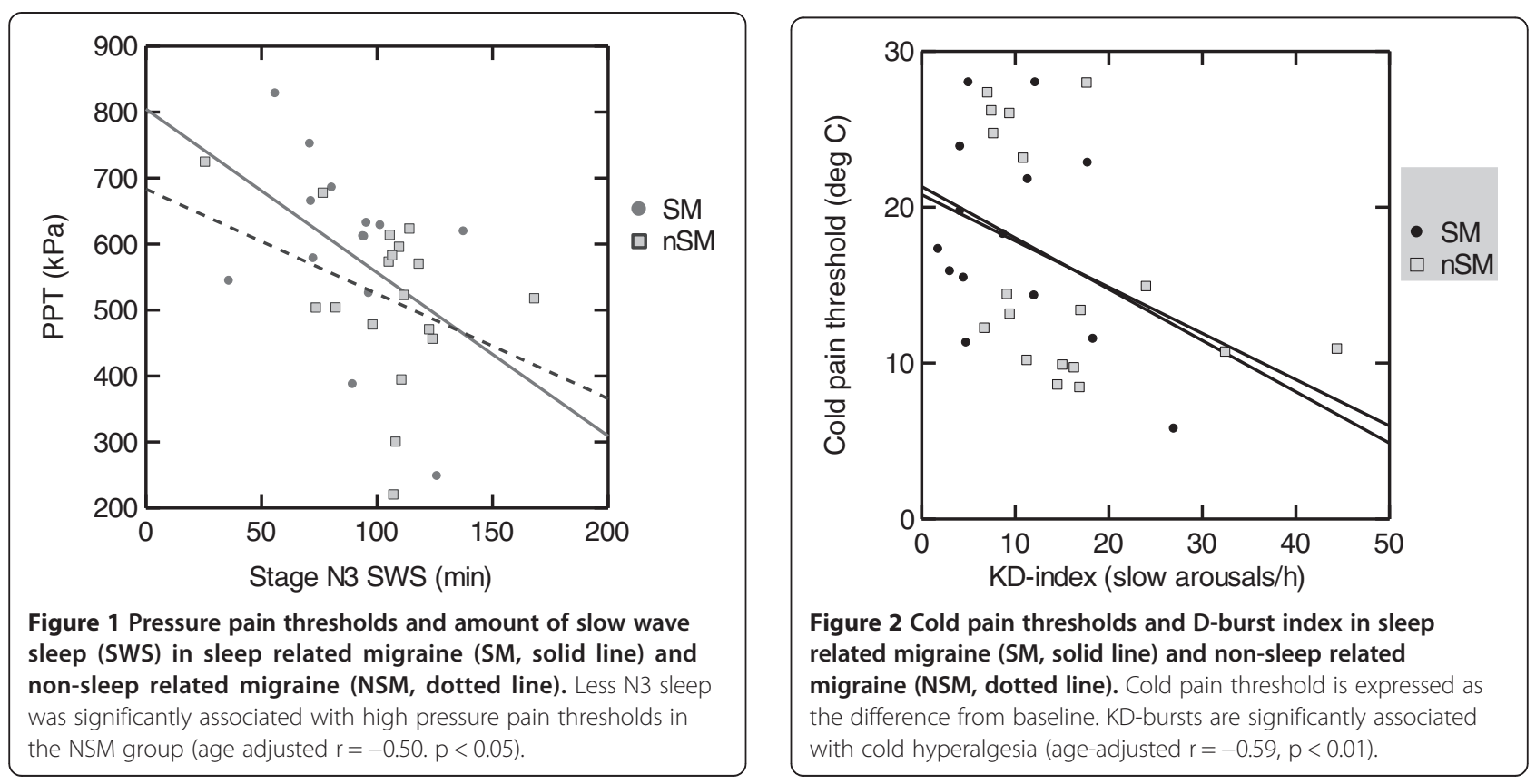
and signs indicating that daytime is more tiring for NSM patients than controls. It can be hypothesized that the lack of response decrement to repeating stimuli (habituation) described among migraineurs [46] might chiefly be a characteristic sign of NSM patients.

The apparently preserved arousability among SM patients might in fact be sign of high resistance towards daytime load consistent with the idea that SM patients do not reach their overload limit before nighttime. The negative effect of high arousability might be an increased vulnerability to reduced sleep quality in SM patients. It is possible that small disturbances in sleep quality (that normally are not perceived as sleep disruptive) are the drops that makes the flood in these patients; i.e. triggering a migraine attack during night. This notion fits with the parallel increase in sleep disturbances and sleep related migraine with age [47] and with the proposal that apnea disrupted sleep also can trigger a migraine attack [48].

The "hypoarousability" of the NSM and robust arousability among SM could in principle be related to the periaqueductal gray matter, a structure which is assumed important in the migraine pathogenesis [49], since "hypoarousal" and "hyperarousal" has been linked to ventrolateral and lateral regions respectively [50].

\section{Strengths and limitations}

The strength of this study is the blinded, controlled, prospective, and population based design, thereby also avoiding hospital-based severe and longstanding migraine cases.

There are different arousal definitions: AASM accepts only fast arousals [32]. Phase of transient activation (PAT) [33] is another definition of fast arousals. Low frequency episodes as D- and K-bursts are found related to temporarily increased heart rate and scored separately as arousals before [33]. The CAP system includes beneficially both fast and slow arousals in one scoring system [51] and is also previously used in examination of sleep among sleepmigraineurs [14]. However, CAP scoring covers only NREM sleep, is quite complicated to score [52] and sleepmigraineurs' sleep is previously also investigated without CAP scoring [15]. In the present exploratory study we included both fast and slow arousals as in the CAP system, but we intended to score both fast and slow arousals separately, without considering sleep phase and the time interval between the different arousal episodes.

Only one PSG recording from each patient in the present study excluded the possibility to evaluate intra individual changes in different migraine phases. A possible first night effect on slow D- and K-arousal bursts and fast microarousal bursts was non-significant and very small in one study [53]. Besides, any other systematic first nighteffects would probably affect groups in a similar way and accordingly be cancelled out in a statistical comparison. Our method for separating migraineurs into SM- and NSM patients by one question on the most typical headache onset time is a weakness. A more objective way would be to evaluate headache onset in diaries for several months. However, as we only had diaries for four weeks, this alternative was not possible in the present study.

Furthermore no corrections for multiple comparisons were done because the study was exploratory, and we did not want to increase type II failures on the cost of reducing type I failures [36,54]. However, the chosen approach does increase the risk of false positives (type I errors) and our findings should accordingly be independently reproduced before firm conclusions can be drawn.

\section{Conclusion}

In conclusion, in this first prospective exploratory casecontrol study evaluating sleep in interictal SM- and NSM patients and controls, we found small, but probably important differences. NSM patients showed a sleep pattern consistent with foregoing sleep deprivation even if sleep times in sleep diaries were normal. SM patients on the other hand, had signs of slightly disturbed sleep. As far as we know, no others have compared PSG-sleep between NSM and controls. However, our results need independent confirmation.

\section{Competing interest}

The authors declare that they have no competing interest.

\section{Authors' contribution}

ME mounted some PSGs and performed some pain threshold measurements, analyzed all PSGs, performed the statistical analysis, prepared the initial draft and was the main author of the present manuscript. KH included patients in the study. GG was contact person for the participants, handled and typed all questionnaires. TS had the original idea of the study; he has made all the data files for statistics and been the main supervisor in all processes. All authors have contributed to the practical plans for the study, read, revised and approved the final manuscript.

\section{Acknowledgements}

We thank Lars Jacob Stovner for diagnosing some of the migraineurs included in this study and both Marit Stjern and Grethe Helde for practical assistance. This study was supported by grant from Department of clinical neuroscience Clinical Neurosciences; Norwegian University of Science and Technology, and Liaison Committee between the Central Norway Regional Health Authority (RHA) and the Norwegian University of Science and Technology (NTNU). Trondheim, Norway.

\section{Author details}

'Department of Clinical Neurosciences, PB 8905, MTFS, Norwegian University of Science and Technology, Trondheim N-7489, Norway. ${ }^{2}$ Department of Neurology and Clinical Neurophysiology, St. Olavs Hospital, Trondheim N-7006, Norway. ${ }^{3}$ Norwegian National Headache Centre, St. Olavs Hospital, Trondheim N-7006, Norway. ${ }^{4}$ Department of Neurology, Haukeland University Hospital, Bergen, Norway. ${ }^{5}$ Department of Clinical Medicine, University of Bergen, Bergen N-5021, Norway. 


\section{References}

1. Gori S, Morelli N, Maestri M, Fabbrini M, Bonanni E, Murri L (2005) Sleep quality, chronotypes and preferential timing of attacks in migraine without aura. J Headache Pain 6(4):258-260

2. Kelman L, Rains JC (2005) Headache and sleep: examination of sleep patterns and complaints in a large clinical sample of migraineurs. Headache 45(7):904-910

3. Olesen J (2004) The International Classification of Headache Disorders: 2nd edition. Cephalalgia 24(Suppl 1):9-160

4. Kelman $L$ (2007) The triggers or precipitants of the acute migraine attack. Cephalalgia 27(5):394-402

5. Andress-Rothrock D, King W, Rothrock J (2010) An analysis of migraine triggers in a clinic-based population. Headache 50(8):1366-1370

6. American Academy of Sleep Medicine (2005) International classification of sleep disorders 2nd edition: Diagnostic and coding manual, 2nd edn. American Academy of Sleep Medicine, Westchester, Illinois

7. Rains JC (2011) Morning headache in habitual snorers. Cephalalgia 31(9):981-983

8. Ulfberg J, Carter N, Talback M, Edling C (1996) Headache, snoring and sleep apnoea. J Neurol 243(9):621-625

9. Alstadhaug K, Salvesen R, Bekkelund S (2007) Insomnia and circadian variation of attacks in episodic migraine. Headache 47(8):1184-1188

10. Odegard SS, Engstrom M, Sand T, Stovner L, Zwart JA, Hagen K (2010) Associations between sleep disturbance and primary headaches: the third Nord-Trondelag Health Study. J Headache Pain 11(3):197-206

11. Dodick DW, Eross EJ, Parish JM, Silber M (2003) Clinical, anatomical, and physiologic relationship between sleep and headache. Headache 43(3):282-292

12. Paiva T, Batista A, Martins P, Martins A (1995) The relationship between headaches and sleep disturbances. Headache 35(10):590-596

13. Holzhammer J, Wober C (2006) Non-alimentary trigger factors of migraine and tension-type headache. Schmerz 20(3):226-237

14. Della Marca G, Vollono C, Rubino M, Di Trapani G, Mariotti P, Tonali PA (2006) Dysfunction of arousal systems in sleep-related migraine without aura. Cephalalgia 26(7):857-864

15. Goder R, Fritzer G, Kapsokalyvas A, Kropp P, Niederberger U, Strenge H, Gerber WD, Aldenhoff JB (2001) Polysomnographic findings in nights preceding a migraine attack. Cephalalgia 21(1):31-37

16. Kristiansen HA, Kvaerner K, Akre H, Overland B, Russell MB (2010) Migraine and sleep apnea in the general population. J Headache Pain 12(1):55-61

17. Azevedo E, Manzano GM, Silva A, Martins R, Andersen ML, Tufik S (2011) The effects of total and REM sleep deprivation on laser-evoked potential threshold and pain perception. Pain 152(9):2052-2058

18. Roehrs T, Hyde M, Blaisdell B, Greenwald M, Roth T (2006) Sleep loss and REM sleep loss are hyperalgesic. Sleep 29(2):145-151

19. Kundermann B, Spernal J, Huber MT, Krieg JC, Lautenbacher S (2004) Sleep deprivation affects thermal pain thresholds but not somatosensory thresholds in healthy volunteers. Psychosom Med 66(6):932-937

20. Onen SH, Alloui A, Gross A, Eschallier A, Dubray C (2001) The effects of total sleep deprivation, selective sleep interruption and sleep recovery on pain tolerance thresholds in healthy subjects. J Sleep Res 10(1):35-42

21. Smith MT, Edwards RR, McCann UD, Haythornthwaite JA (2007) The effects of sleep deprivation on pain inhibition and spontaneous pain in women. Sleep 30(4):494-505

22. Sand T, Zhitniy N, Nilsen KB, Helde G, Hagen K, Stovner LJ (2008) Thermal pain thresholds are decreased in the migraine preattack phase. Eur J Neurol 15(11):1199-1205

23. Engstrom M, Hagen K, Bjork MH, Stovner LJ, Gravdahl GB, Stjern M, Sand T (2013) Sleep quality, arousal and pain thresholds in migraineurs: a blinded controlled polysomnographic study. J Headache Pain 14(1):12

24. Johns MW (1991) A new method for measuring daytime sleepiness: the Epworth sleepiness scale. Sleep 14(6):540-545

25. Miletin MS, Hanly PJ (2003) Measurement properties of the Epworth sleepiness scale. Sleep Med 4(3):195-199

26. Engstrøm M, Ødegård SS, Sand T, Stovner L, Zwart JA, Hagen K (2011) The reliability of a New Sleep Screening Questionnaire for Large PopulationBased Studies: The Third Nord-Trøndelag Health Study The Open Sleep Journal 4:14-19

27. Buysse DJ, Reynolds CF 3rd, Monk TH, Berman SR, Kupfer DJ (1989) The Pittsburgh Sleep Quality Index: a new instrument for psychiatric practice and research. Psychiatry Res 28(2):193-213

28. Zigmond AS, Snaith RP (1983) The hospital anxiety and depression scale. Acta Psychiatr Scand 67(6):361-370
29. Trenkwalder C, Paulus W, Walters AS (2005) The restless legs syndrome. Lancet neurology 4(8):465-475

30. Klem GH, Luders HO, Jasper HH, Elger C (1999) The ten-twenty electrode system of the International Federation. The International Federation of Clinical Neurophysiology. Electroencephalogr Clin Neurophysiol Suppl 52:3-6

31. American Academy of Sleep Medicine Task Force Sleep-related breathing disorders in adults: recommendations for syndrome definition and measurement techniques in clinical research. Sleep 22(5):667-689

32. American Academy of Sleep Medicine W (2007) The AASM Manual for the Scoring of Sleep and Associated Events. Rules, Terminology and Technical Specifications. American Academy of Sleep Medicine. Westchester, IL 60154, U.S.A

33. Sforza E, Jouny C, Ibanez V (2000) Cardiac activation during arousal in humans: further evidence for hierarchy in the arousal response. Clin Neurophysiol 111(9):1611-1619

34. Parrino L, Boselli M, Spaggiari MC, Smerieri A, Terzano MG (1998) Cyclic alternating pattern (CAP) in normal sleep: polysomnographic parameters in different age groups. Electroencephalogr Clin Neurophysiol 107(6):439-450

35. Halasz P, Terzano M, Parrino L, Bodizs R (2004) The nature of arousal in sleep. J Sleep Res 13(1):1-23

36. Perneger TV (1998) What's wrong with Bonferroni adjustments. Bmj 316 (7139):1236-1238

37. Nicholas CL, Trinder J, Colrain IM (2002) Increased production of evoked and spontaneous K-complexes following a night of fragmented sleep. Sleep 25(8):882-887

38. De Gennaro L, Ferrara M, Bertini M (2001) EEG arousals in normal sleep: variations induced by total and selective slow-wave sleep deprivation. Sleep 24(6):673-679

39. Youngberg MR, Karpov IO, Begley A, Pollock BG, Buysse DJ (2011) Clinical and physiological correlates of caffeine and caffeine metabolites in primary insomnia. J Clin Sleep Med 7(2):196-203

40. Sand T (1991) EEG in migraine: a review of the literature. Funct Neurol 6(1):7-22

41. Terzano MG, Parrino L, Smerieri A, Carli F, Nobili L, Donadio S, Ferrillo F (2005) CAP and arousals are involved in the homeostatic and ultradian sleep processes. J Sleep Res 14(4):359-368

42. Cortelli P, Pierangeli G, Montagna P (2010) Is migraine a disease? Neurol Sci 31(Suppl 1):S29-31

43. Zwart JA, Dyb G, Hagen K, Odegard KJ, Dahl AA, Bovim G, Stovner L (2003) Depression and anxiety disorders associated with headache frequency. The Nord-Trondelag Health Study. Eur J Neurol 10(2):147-152

44. Lanteri-Minet M, Radat F, Chautard MH, Lucas C (2005) Anxiety and depression associated with migraine: influence on migraine subjects' disability and quality of life, and acute migraine management. Pain 118(3):319-326

45. Porkka-Heiskanen T, Kalinchuk AV (2011) Adenosine, energy metabolism and sleep homeostasis. Sleep Med Rev 15(2):123-135

46. Coppola G, Pierelli F, Schoenen J (2009) Habituation and migraine. Neurobiol Learn Mem 92(2):249-259

47. Gori S, Lucchesi C, Morelli N, Maestri M, Bonanni E, Murri L (2012) Sleep-related migraine occurrence increases with aging. Acta Neurol Belg 112(2):183-187

48. Johnson KG, Ziemba AM, Garb JL (2012) Improvement in Headaches With Continuous Positive Airway Pressure for Obstructive Sleep Apnea: A Retrospective Analysis. Headache 53(2):333-343

49. Goadsby PJ (2005) Migraine pathophysiology. Headache 45(Suppl 1):S14-24

50. Keay KA, Bandler R (2001) Parallel circuits mediating distinct emotional coping reactions to different types of stress. Neurosci Biobehav Rev 25(7-8):669-678

51. Terzano MG, Parrino L (2000) Origin and Significance of the Cyclic Alternating Pattern (CAP). REVIEW ARTICLE. Sleep Med Rev 4(1):101-123

52. Thomas RJ (2007) Cyclic alternating pattern in the electroencephalogram: what is its clinical utility? Sleep 30(5):553-555

53. Sforza E, Chapotot F, Pigeau R, Buguet A (2008) Time of night and first night effects on arousal response in healthy adults. Clin Neurophysiol 119(7):1590-1599

54. Schulz KF, Grimes DA (2005) Multiplicity in randomised trials I: endpoints and treatments. Lancet 365(9470):1591-1595

\section{doi:10.1186/1129-2377-14-68}

Cite this article as: Engstrøm et al.: Sleep-related and non-sleep-related migraine: interictal sleep quality, arousals and pain thresholds. The Journal of Headache and Pain 2013 14:68. 